\title{
Preliminary DRIS Diagnostic Norms for Pecan
}

R.B. Beverly

Department of Horticulture, Georgia Experiment Station, Griffin, GA 30223

\section{R.E. Worley}

Department of Horticulture, Coastal Plain Experiment Station, Tifton, GA 31793

Additional index words. Carya illinoinensis, Diagnosis and Recommendation Integrated System

The Diagnosis and Recommendation Integrated System (DRIS) uses nutrient relationships, such as concentration ratios in tissue, to assess nutrient balance and diagnose the nutrient most likely to limit growth and yield (Beverly, 1991; Walworth and Sumner, 1987). Based on field fertility research with pecan [Carya illinoinensis (Wangenh.) K. Koch] (R.E.W., unpublished data), we compiled more than 3000 observations, including yield and leaf concentrations of 11 essential nutrients and $\mathrm{Al}$ (Table 1). The sampling and analytical techniques have been published (Worley, 1974). The means and cvs of element concentrations (Table 1) and ratios (Table 2) corresponding to yields $\geq 58 \mathrm{~kg} /$ tree (the 75 th percentile by yield) can be used to calculate DRIS index values (Beverly, 1991; Walworth and Sumner, 1987). Since alternative forms of expression (e.g., $\mathrm{N}: \mathrm{P}$ vs. P : N) should not affect diagnostic accuracy, the ratios presented are based on the arbitrary order of concentrations in Table 1 . We were unable to identify a suitable independent population from the literature with which to

Received for publication 15 Apr. 1991. Accepted for publication 28 Oct. 1991. The cost of publishing this paper was defrayed in part by the payment of page charges. Under postal regulations, this paper therefore must be hereby marked advertisement solely to indicate this fact. test the diagnostic accuracy of the norms; thus, we consider the norms preliminary.

\section{Literature Cited}

Beverly, R.B. 1991. A practical guide to the Diagnosis and Recommendation Integrated System (DRIS). Micro-Macro Publishing, Athens, Ga.

Walworth, J.L. and M.E. Sumner. 1987. The diagnosis and recommendation integrated system (DRIS). Adv. Soil. Sci. 6:149-188.

Worley. R.E. 1974. Effect of N, P, K, and lime on yield, nut quality, tree growth, and leaf analysis of pecan (Carya illinoinensis W.). J. Amer. Soc. Hort. Sci. 99:49-57.

Table 1. Leaf element concentrations in pecan trees with yield $\geq 58 \mathrm{~kg} /$ tree (75th yield percentile).

\begin{tabular}{lccr}
\hline \hline Element $^{z}$ & $\mathrm{n}$ & Mean & $\mathrm{CV}$ \\
\hline $\mathrm{N}$ & 585 & 27.2 & 9.4 \\
$\mathrm{P}$ & 641 & 1.40 & 18.1 \\
$\mathrm{~K}$ & 641 & 10.2 & 20.9 \\
$\mathrm{Ca}$ & 641 & 14.5 & 33.4 \\
$\mathrm{Mg}$ & 641 & 3.82 & 29.9 \\
$\mathrm{Fe}$ & 487 & 89.4 & 40.9 \\
$\mathrm{Mn}$ & 596 & 324 & 54.5 \\
$\mathrm{Zn}$ & 641 & 126 & 60.0 \\
$\mathrm{Cu}$ & 401 & 9.69 & 32.4 \\
$\mathrm{Mo}$ & 134 & 6.30 & 28.1 \\
$\mathrm{~B}$ & 274 & 40.1 & 34.7 \\
$\mathrm{Al}$ & 273 & 1380 & 34.3 \\
\hline
\end{tabular}

${ }^{\mathrm{x}}$ Nitrogen, $\mathrm{P}, \mathrm{K}, \mathrm{Ca}$, and $\mathrm{Mg}$ expressed in $\mathrm{g} \cdot \mathrm{kg}^{-1}$; other elements in $\mathrm{mg} \cdot \mathrm{kg}^{-1}$.
Table 2. Leaf element concentration ratios in pecan trees with yield $\geq 58 \mathrm{~kg} /$ tree (75th yield percentile).

\begin{tabular}{|c|c|c|}
\hline Ratio $^{z}$ & Mean & $\mathrm{CV}$ \\
\hline $\mathrm{N}: \mathrm{P}$ & 19.8 & 18.2 \\
\hline $\mathrm{N}: \mathrm{K}$ & 2.74 & 20.9 \\
\hline $\mathrm{N}: \mathrm{Ca}$ & 2.44 & 130 \\
\hline $\mathrm{N}: \mathrm{Mg}$ & 7.74 & 36.7 \\
\hline $\mathrm{N}: \mathrm{Fe}$ & 0.349 & 36.4 \\
\hline $\mathrm{N}: \mathrm{Mn}$ & 0.107 & 47.4 \\
\hline $\mathrm{N}: \mathrm{Zn}$ & 0.306 & 76.5 \\
\hline $\mathrm{N}: \mathrm{Cu}$ & 3.13 & 41.0 \\
\hline $\mathrm{N}: \mathrm{Mo}$ & 4.56 & 28.0 \\
\hline $\mathrm{N}: \mathrm{B}$ & 0.746 & 40.2 \\
\hline $\mathbf{N}: \mathrm{Al}$ & 0.0257 & 138 \\
\hline$P: K$ & 0.143 & 25.4 \\
\hline $\mathrm{P}: \mathrm{Ca}$ & 0.123 & 117 \\
\hline $\mathrm{P}: \mathrm{Mg}$ & 0.414 & 46.5 \\
\hline $\mathrm{P}: \mathrm{Fe}$ & 0.0175 & 32.0 \\
\hline $\mathrm{P}: \mathrm{Mn}$ & 0.00535 & 41.3 \\
\hline$P: Z n$ & 0.0164 & 76.4 \\
\hline $\mathrm{P}: \mathrm{Cu}$ & 0.163 & 38.9 \\
\hline P : Mo & 0.301 & 25.6 \\
\hline$P: B$ & 0.0412 & 40.0 \\
\hline $\mathrm{P}: \mathrm{Al}$ & 0.00143 & 154 \\
\hline $\mathrm{K}: \mathrm{Ca}$ & 0.948 & 151 \\
\hline $\mathrm{K}: \mathrm{Mg}$ & 2.97 & 46.3 \\
\hline $\mathrm{K}: \mathrm{Fe}$ & 0.134 & 35.9 \\
\hline $\mathrm{K}: \mathrm{Mn}$ & 0.0409 & 50.7 \\
\hline $\mathrm{K}: \mathrm{Zn}$ & 0.121 & 77.3 \\
\hline $\mathrm{K}: \mathrm{Cu}$ & 1.23 & 41.7 \\
\hline K : Mo & 1.86 & 32.4 \\
\hline $\mathbf{K}: \mathbf{B}$ & 0.319 & 53.6 \\
\hline $\mathrm{K}: \mathrm{Al}$ & 0.0104 & 137 \\
\hline $\mathrm{Ca}: \mathrm{Mg}$ & 4.13 & 42.0 \\
\hline $\mathrm{Ca}: \mathrm{Fe}$ & 0.193 & 54.2 \\
\hline $\mathrm{Ca}: \mathrm{Mn}$ & 0.0583 & 58.5 \\
\hline $\mathrm{Ca}: \mathrm{Zn}$ & 0.168 & 75.4 \\
\hline $\mathrm{Ca}: \mathrm{Cu}$ & 1.62 & 47.8 \\
\hline $\mathrm{Ca}: \mathrm{Mo}$ & 2.27 & 29.2 \\
\hline $\mathrm{Ca}: \mathrm{B}$ & 0.325 & 41.6 \\
\hline $\mathrm{Ca}: \mathrm{Al}$ & 0.0109 & 135 \\
\hline $\mathrm{Mg}: \mathrm{Fe}$ & 0.0518 & 45.2 \\
\hline $\mathrm{Mg}: \mathrm{Mn}$ & 0.0158 & 58.3 \\
\hline $\mathrm{Mg}: \mathrm{Zn}$ & 0.0438 & 71.9 \\
\hline $\mathrm{Mg}: \mathrm{Cu}$ & 0.470 & 49.4 \\
\hline $\mathrm{Mg}: \mathrm{Mo}$ & 0.565 & 42.9 \\
\hline $\mathrm{Mg}: \mathrm{B}$ & 0.111 & 45.0 \\
\hline $\mathrm{Mg}: \mathrm{Al}$ & 0.00389 & 155 \\
\hline $\mathrm{Fe}: \mathrm{Mn}$ & 0.354 & 46.0 \\
\hline $\mathrm{Fe}: \mathrm{Zn}$ & 1.04 & 91.7 \\
\hline $\mathrm{Fe}: \mathrm{Cu}$ & 9.98 & 42.9 \\
\hline $\mathrm{Fe}: \mathrm{Mo}$ & 18.6 & 45.8 \\
\hline $\mathrm{Fe}: \mathrm{B}$ & 2.77 & 46.5 \\
\hline $\mathrm{Fe}: \mathrm{Al}$ & 0.0958 & 149 \\
\hline $\mathrm{Mn}: \mathrm{Zn}$ & 3.23 & 83.6 \\
\hline $\mathrm{Mn}: \mathrm{Cu}$ & 35.4 & 56.6 \\
\hline $\mathrm{Mn}: \mathrm{Mo}$ & 70.7 & 45.3 \\
\hline $\mathrm{Mn}: \mathrm{B}$ & 10.2 & 65.0 \\
\hline $\mathrm{Mn}: \mathrm{Al}$ & 0.328 & 171 \\
\hline $\mathrm{Zn}: \mathrm{Cu}$ & 14.8 & 57.6 \\
\hline $\mathrm{Zn}: \mathrm{Mo}$ & 21.5 & 54.7 \\
\hline $\mathrm{Zn}: \mathrm{B}$ & 3.69 & 66.5 \\
\hline $\mathrm{Zn}: \mathrm{Al}$ & 0.125 & 183 \\
\hline $\mathrm{Cu}: \mathrm{Mo}$ & 1.70 & 41.1 \\
\hline $\mathrm{Cu}: \mathrm{B}$ & 0.276 & 43.8 \\
\hline $\mathrm{Cu}: \mathrm{Al}$ & 0.00954 & 148 \\
\hline Mo : B & 0.183 & 71.5 \\
\hline Mo : Al & 0.00586 & 159 \\
\hline $\mathrm{B}: \mathrm{Al}$ & 0.0384 & 170 \\
\hline
\end{tabular}

